\title{
Incorporación, uso y apropiación social de las TIC para una educación de calidad
}

\section{Incorporation, use and social appropriation of ICT for quality education}

DOI: http://dx.doi.org/10.17981/cultedusoc.12.1.2021.04

Recibido: 14 de abril de 2020 Aceptado:04 de septiembre de 2020 Publicado: 22 de noviembre de 2020

\author{
María Estefanía Orozco-García \\ Fundación Universitaria de Popayán. Popayán (Colombia) \\ maria.orozco@docente.fup.edu.co \\ Fredy Eduardo Vásquez-Rizo \\ Universidad Autónoma de Occidente. Cali (Colombia) \\ fvasquez@uao.edu.co \\ Jesús Gabalán-Coello \\ Universidad Católica de Pereira. Pereira (Colombia) \\ jesus.gabalan@ucp.edu.co
}

Para citar este artículo:

Orozco-García, M., Vásquez-Rizo, F. y Gabalán-Coello, J. (2021). Incorporación, uso y apropiación social de las TIC para una educación de calidad. Cultura, Educación y Sociedad, 12(1), 19-62. DOI: http://dx.doi.org/10.17981/cultedusoc.12.1.2021.04

\section{Resumen}

El gobierno colombiano ha implementado la estrategia Plan Vive Digital como forma de articular las poblaciones rurales al desarrollo tecnológico. Se presenta el análisis de dicha política en el municipio de El Tambo (Cauca, Colombia), con la intención de determinar su efectividad desde la comprensión del uso y apropiación de la tecnología, localizada en una institución educativa oficial. Desde un enfoque multimétodo, de carácter mixto, se trabajan tres fases: caracterización contextual, análisis de usos y prácticas de apropiación tecnológica y planteamiento de estrategia de mejora. Así, se logra establecer que la infraestructura empleada no es adecuada, que el capital humano no está conforme con la efectividad de los procesos tecnológicos y que el proceso de inclusión digital no es efectivo, lo que lleva a proponer una estrategia formativa-tecnológica para promover una mayor inserción, desde la alfabetización digital y la reducción de la brecha social. Todo esto para concluir que es plausible la intención estatal, pero no es suficiente, ya que se implanta la estrategia, pero se desconocen factores relevantes que inciden en su correcto desarrollo, situación que perpetúa la inequidad.

Palabras clave: Apropiación social de las TIC; alfabetización digital; educación para el desarrollo; brecha digital; tecnología educacional

\section{Abstract}

The colombian Government has implemented the Plan Vive Digital strategy as a way of linking rural populations to technological development. The analysis of said policy is presented in the municipality of El Tambo (Cauca, Colombia), with the intention of determining its effectiveness from the understanding of the use and appropriation of technology, located in an official educational institution. From a multimethod approach, of a mixed nature, three phases are worked: contextual characterization, analysis of uses and practices of technological appropriation and proposal of an improvement strategy. Thus, it is possible to establish that the infrastructure used is not adequate, that human capital is not satisfied with the effectiveness of technological processes and that the process of digital inclusion is not effective, which leads to proposing a training-technological strategy to promote greater insertion, from digital literacy and the reduction of the social gap. All this to conclude that the state intention is plausible, but it is not enough, since the strategy is implemented, but relevant factors that affect its correct development are unknown, a situation that perpetuates inequity.

Keywords: Social appropriation of ICT; digital literacy; development education; digital gap; educational technology 
La implementación y uso de las Tecnologías de la Información y la Comunicación-TIC en la sociedad actual, conocida como Sociedad de la Información y el Conocimiento (Escobar, 2014), se han ido incrementando de forma exponencial. Prueba de ello es que la Organización de las Naciones Unidas (ONU, 2019) considera que las TIC son hoy el medio por el cual las poblaciones tienen la posibilidad de vencer sus diferencias sociales y fortalecer su economía, a partir de la reducción de su brecha digital.

Sin embargo, esta reducción no funciona de la misma manera en todos los contextos, ya que son dependientes del diseño y puesta en marcha de efectivas políticas públicas de desarrollo (Barraquero, 2006; 2009; Chong, 2011), en su mayoría propiciadas por instancias de vanguardia, que defienden sus propios intereses y que dejan la implementación de dichas políticas a merced de directrices que poco se preocupan por disminuir las desigualdades existentes (Sachs, 1996; Barraquero y Ángel, 2014).

Por ello, es necesario que se entienda que tales directrices para el fomento, implementación y uso de TIC deben estar acompañadas de posibilidades reales de desarrollo y, primordialmente, con opción de acceso a la población (Echeverría, 2008). Y es justamente esta situación la que lleva a la realización del presente documento, el cual intenta determinar la efectividad de una de esas políticas de desarrollo: el Plan Vive Digital, orientado por el Gobierno colombiano, teniendo como epicentro de estudio el municipio de El Tambo (Cauca, Colombia), cuyo punto principal se encuentra en una institución educativa del medio rural.

El plan que tiene como objetivo impulsar la masificación del acceso y uso de internet en la región (Ministerio de las Tecnologías de la Información y las Comunicaciones-MinTIC, 2009; Paulhiac y Ortega, 2019), creando para ello un ecosistema digital soportado en cuatro ejes fundamentales: usuarios, infraestructura, aplicaciones y servicios (MinTIC, 2018), los cuales se corresponden con los pilares de la gestión de la información y el conocimiento (Vásquez-Rizo y Gabalán-Coello, 2011): capital humano (usuarios), capital estructural (infraestructura y aplicaciones) y capital relacional (servicios).

Con el presente artículo se pretende aportar a la comprensión de las distintas dinámicas, prácticas culturales y representaciones sociales mediadas por TIC en la vida de los pobladores de este contexto rural, a partir del análisis de la efectividad de las políticas públicas implementadas y la propuesta final de una estrategia tecnológica-formativa que permita fortalecer la relación entre el punto y sus usuarios.

\section{REVISIÓN DE LA LITERATURA}

Las TIC han propiciado nuevos espacios de resignificación y potencialización (Cobo, 2009) y las regiones periféricas no son ajenas a esta influencia, por ello, deben ser tenidas en cuenta en las planificaciones relacionadas con el tema. Es por esto que los gobiernos se han visto en la necesidad de orientar estrategias de desarrollo hacia la conectividad y el cumplimiento de los Objetivos del Desarrollo Sostenible-ODS en estas zonas (Comisión Económica para América Latina y el Caribe-CEPAL, 2018; Programa de las Naciones Unidas para el Desarrollo-PNUD, 2020), con miras a reducir 
la desigualdad, propiciada por las diferencias en los avances tecnológicos, la brecha digital y su disímil incorporación en el paradigma de la Sociedad de la Información y el Conocimiento.

En Colombia, el MinTIC es el encargado de velar porque este escenario suceda, así como promover y potenciar la realidad digital en toda la población. Es así como el Plan Vive Digital surge como un mecanismo nacional para reducir dichas falencias, a través de la masificación y democratización del uso y apropiación de internet y las TIC (MinTIC, 2020).

Sin embargo, pese a este propósito loable del Estado, los cometidos de esta estrategia aún son incipientes en algunos escenarios, pues en la práctica la cobertura e infraestructura tecnológica aún tienen que luchar contra las precarias condiciones de estos territorios, en muchos casos carentes de los recursos fundamentales, lo que impide llevar a buen término lo planteado desde la planificación, ocasionando la continuidad de su estado de exclusión (PNUD Colombia, 2015).

A esto se suman las limitaciones existentes, producto en algunos casos de las complejas condiciones topográficas, situación que también afecta la transformación tecnológica y deja estos territorios a merced de acciones de ilegalidad y violencia, lo que causa graves problemas sociales, mucho más prioritarios que los tecnológicos.

Tal es el caso del municipio de El Tambo (Cauca), un territorio ubicado en el suroccidente colombiano, de población principalmente mestiza, afrodescendiente e indígena, con vocación campesina, distribuida en una cabecera municipal, 19 corregimientos, 215 veredas y dos resguardos indígenas (Departamento Administrativo Nacional de Estadística-DANE, 2018; 2019). Sujetos que se desplazan desde grandes distancias, y a través de innumerables riesgos sociales, a la cabecera municipal donde está el principal punto Vive Digital (MinTIC, 2017), el cual alberga alrededor de 480 estudiantes (la mayoría de estrato 1, todos nativos o semi-nativos digitales (Prensky, 2001), quienes tienen que hacer uso compartido de 23-25 equipos de cómputo y un acceso intermitente a internet (recursos ausentes en gran parte del municipio).

En la actualidad, las condiciones para la expansión tecnológica no están dadas en muchos de estos escenarios (Lemus, 2017), donde la tecnología sigue siendo obsoleta, la cobertura de internet precaria y el analfabetismo digital evidente, por la cual las estrategias de subsanación deben estar acompañadas de planes de apropiación y educación en TIC que posibiliten una verdadera inclusión social y que garanticen el bienestar de los territorios y sus individuos (Vásquez, 2012), enfatizando en una real transformación y no en el simple cumplimiento de indicadores de gestión que responden a intereses externos a las poblaciones (Pathak-Shelat \& DeShano, 2014).

De esta forma, se avanza en dos frentes: uno relacionado con la alfabetización digital de los pueblos (Bawden, 2002), y otro relacionado con el reconocimiento de su contexto y la conservación de sus identidades autóctonas (Chong, Aguilar, Ornelas, Morales y Torre, 2011), pues es fundamental crecer en lo tecnológico, pero sin que este crecimiento esté sujeto incondicionalmente al ritmo vertiginoso impuesto por la sociedad de la información y el conocimiento, que muchas veces desconoce la idiosincrasia de los territorios emergentes (Villacrés, 2016). 
Por lo tanto, se puede inferir que el éxito de la apropiación social de las TIC no consiste solamente en llevar tecnología a las zonas marginadas (Calderón y Acuña, 2017), pues esto debe ir más allá del fortalecimiento del capital estructural, sino en promover el conocimiento y el aprendizaje de las personas (capital humano) (Chaparro, 2003), así como garantizar un efectivo y útil relacionamiento entre éstas y las TIC (capital relacional) (Cabrera, 2006).

Tarea que se torna mucho más compleja cuando son las nuevas generaciones (como es el caso de los alumnos de la institución objeto de estudio) quienes deben ser conscientes de esta situación, las cuales por su condición de juventud están conformadas por sujetos fácilmente descrestados por las tecnologías de vanguardia, dejando desatendidas, muchas veces, las afectaciones que dicho seguimiento incondicional y afán por no quedar relegados pueden ocasionar en la transformación de su identidad cultural (Castells, 2009; Quinchoa, 2011; Morduchowicz, 2013; Paz, 2015; Andrade, Carbonell y López, 2018).

Todo lo anterior sugiere que, el desarrollo de una comunidad no se debe limitar a la posibilidad de tener o no conectividad o tecnología (Barón y Gómez, 2014), sino que se debe ir mucho más lejos, al pensar en los intereses y necesidades de los sujetos a los que les va a servir; es decir, el énfasis debe estar en que la apropiación de las TIC debe poseer un carácter utilitario y pertinente, o sea un carácter al servicio de lo social (Rueda, 2005; Winocur, 2013). Solo así se logrará la prosperidad económica, pero con equidad (Escobar, 2003; Gumucio, 2011), y la reducción real de la brecha digital (Espinoza, 2010; Fernández, 2012), y que éstas sirvan para el avance específico de la comunidad y sus miembros.

\section{Metodología}

Esta investigación se realiza dentro de los parámetros de un enfoque multimétodo, de naturaleza descriptiva, explicativa, de análisis y propositiva, en el marco de la investigación mixta (cualitativa y cuantitativa) (Méndez, 2015; Hernández, Fernández y Baptista, 2015), a partir de la caracterización de los usos y prácticas de apropiación social de las TIC en un escenario concreto: el punto Vive Digital en una institución educativa oficial ubicada en El Tambo (Cauca).

Para ello, se emplean la observación, la encuesta (cuyos componentes del diseño fueron abordados teniendo una fase de validación por expertos y validación estadística, que permitió decantar las variables objeto de análisis, evitando aspectos inherentes a la colinealidad entre las mismas) y la entrevista semiestructurada como técnicas complementarias de recolección y comparación de datos (no estandarizados), con la intención de analizar las percepciones, emociones y experiencias de los sujetos de estudio en relación con su entorno digital. Por lo tanto, este artículo no pretende medir de manera exclusivamente cuantitativa los alcances del Plan Vive Digital, sino comprender qué tan efectiva ha sido su implementación, desde el entendimiento de los usos y prácticas de apropiación social de las TIC de la población. La Tabla 1 expone las generalidades de dichas técnicas. 
TABLA 1.

Generalidades de las técnicas de investigación

\begin{tabular}{lll}
\hline $\begin{array}{c}\text { Técnica de } \\
\text { investigación }\end{array}$ & \multicolumn{1}{c}{ ¿Qué se busca mediante esta técnica? } & $\begin{array}{c}\text { ¿Qué actores hacen } \\
\text { parte de la indagación? }\end{array}$ \\
\hline Observación & $\begin{array}{l}\text { Caracterizar el contexto TIC del Plan Vive Digital, implementado } \\
\text { en la Institución. }\end{array}$ & $\begin{array}{l}\text { Estudiantes de la } \\
\text { Encuesta }\end{array}$ \\
& $\begin{array}{l}\text { Caracterizar y "medir" generalidades acerca de la apropiación del } \\
\text { Plan, y revisar su implementación. }\end{array}$ & $\begin{array}{l}\text { Institución, como } \\
\text { representantes de un } \\
\text { contexto rural, apoyado }\end{array}$ \\
& $\begin{array}{l}\text { Profundizar en las experiencias observadas y en las respuestas de } \\
\text { la encuesta, y contrastar los objetivos del estudio con la información } \\
\text { Entrevista }\end{array}$ & $\begin{array}{l}\text { en el Plan Vive Digital. } \\
\end{array}$ \\
\hline
\end{tabular}

Fuente: Elaboración propia.

Para la selección de los sujetos de la investigación, se establece un muestreo no probabilístico por conveniencia (Patton, 2014). Esto es que, a partir de los 480 estudiantes de la Institución, se selecciona el 100\% de los estudiantes de los grados 9, 10 y 11 (dado que son quienes mejor cuenta pueden dar del avance formativo). De esta manera, se conforma un grupo representativo de 163 estudiantes que sirve como piloto para la generalización del ejercicio.

De esta forma, el desarrollo metodológico consta de tres fases:

1. Caracterización del contexto TIC del Plan Vive Digital: implementado en la Institución Educativa: utilización de bitácoras para registrar lo observado (Martínez, 2007) - el ambiente tecnológico-académico y los sujetos de estudio (capitales Humano y Estructural).

2. Análisis del uso y prácticas de apropiación tecnológica: profundización de lo observado a patir del diseño y aplicación de encuestas y la realización de entrevistas (énfasis en Capital Relacional). Sistematización de datos.

En el caso de la encuesta, cuyo instrumento es el cuestionario aplicado, que consta de cinco (5) ítems principales, distribuidos en diversas preguntas orientadoras desde un enfoque semiestructurado al interior de cada ítem, ésta se centra en aspectos como: uso de internet y TIC, infraestructura, acceso, incorporación en el aula e implementación y uso del punto Vive Digital (apropiación social), para su elaboración se emplean preguntas de selección y de calificación (escalas tipo Likert, dicotómicas, de selección múltiple, de jerarquía).

En la entrevista se aborda la relación de lo observado y encuestado con temáticas como brecha digital (Vásquez, Rodríguez y Gómez, 2019), habitus tecnológico (Casillas y Ramírez, 2018), alfabetización digital (Pérez, 2015) y uso, implementación y apropiación de TIC (Echeverría, 2008), desde el planteamiento de preguntas relacionadas con factores como: acceso, cobertura, frecuencia de uso, dispositivos, espacios de uso, suficiencia, dominio, conocimiento, preferencias, influencias, educación recibida, entre otros. En cuanto a la sistematización procedimental, se advierte que dicha técnica es aplicada a través de grupos focales, centralizados en los estudiantes de los grados 9, 10 y 11 de la Institución objeto de estudio, previa estructuración de las preguntas según lo observado y encuestado, con posibilidad de recurrir a nuevas preguntas conforme el desarrollo de las entrevistas. 


\section{Planteamiento de una estrategia de mejora para el punto principal del Plan Vive Di-} gital de El Tambo (Cauca): Estrategia que surge como propuesta que intenta integrar el devenir académico de los estudiantes y su vinculación tecnológica, con énfasis en un tema de interés comunal, a partir del desarrollo de tres principales competencias: tecnológica, comunicacional y social.

A través de lo anterior, se describen las principales características asociadas al uso de las TIC en la población objeto de estudio, con la intención de caracterizar el contexto actual de la implantación del Plan Vive Digital en el municipio, haciendo hincapié, principalmente, en su desarrollo e implementación, involucrando una estrategia formativa que permita subsanar algunos de los aspectos deficientes y contribuir así al desarrollo social del municipio y sus habitantes.

\section{Resultados}

Los resultados se presentan organizados según las dimensiones de análisis definidas en la metodología, las cuales se desarrollan en virtud de las técnicas de observación, encuesta y entrevista.

La observación inicial realizada permite establecer que el punto Vive Digital de la Institución Educativa seleccioanda cuenta con dos salas de cómputo, una principal, equipada con 15 computadores de mesa, y otra con entre 8 y 10 portátiles (dependiendo de su estado), una impresora y un scanner, interconectados a través de internet. Todos ellos con software y aditamentos para la generación de contenidos de Adobe (Photoshop, Ilustrator, Flash, Audition, Premier) y paquete Office 2014. En este mismo espacio se tiene un estudio de televisión, cámaras fotográficas, cámara de video profesional, diferentes tipos de trípodes, luces colgantes y un fondo croma verde, así como consola de radio y micrófonos.

La mayoría de los estudiantes hacen uso del punto en el curso de Informática, obligándose a ubicarse entre dos o tres por equipo, lo que implica que se dediquen a otras actividades distintas a su formación, situación que evidencia una subutilización inicial del plan, a pesar que se aprecia en ellos destrezas en el manejo de lo tecnológico. Sumado a lo anterior, otro inconveniente observado consiste en que las clases tienen que ser interrumpidas constantemente o canceladas debido a que el internet es inestable o se torna demasiado lento y a que algunas licencias de software están vencidas.

Adicional a esto, parte del cableado y los puertos externos de los equipos de cómputo se encuentran deteriorados, lo que hace que el intercambio de archivos necesariamente deba realizarse a través de internet, supeditándose todo a su funcionamiento. Actividad que tampoco puede desarrollarse desde el hogar porque la mayoría de los estudiantes carencen del recurso; es decir, no es posible dejar tareas, por lo que el proceso formativo se supedita exclusivamente a la clase y a la conectividad del punto.

La población usuaria del punto Vive Digital, a pesar de ser 100\% rural, sigue los mismos patrones y procesos de aculturación citadina (Escosteguy, Bianchini e Ribas, 2018). Esto se afirma debido por cuanto los contenidos utilizados en la actividad formativa son los mismos que afectan a los usuarios de ciudad (redes sociales, juegos, programas de tv, videos), aspectos que, a pesar de las falencias expuestas, motivan a los jóvenes de El 
Tambo a recurrir al punto, donde pueden hacer uso de los dispositivos institucionales y personales.

Cuando se les indaga acerca del uso de estos dispositivos, las redes sociales ocupan el primer lugar. En una escala de 1 a 5, el promedio de calificación obtenido por este ítem es de 3,93, por encima de actividades académicas $(3,57)$ e investigación $(3,19)$, lo que trasgrede la intención original de alfabetización digital asociada a la formación del punto.

En cuanto a la infraestructura y el acceso, el 86,8\% de los encuestados opina que no existe en el punto una completa suficiencia en relación con sus necesidades y los dispositivos tecnológicos presentes. Sumado a esto, el 89,9\% manifieta no sentirse completamente a gusto con la disponibilidad, velocidad, estabilidad y/o eficiencia del acceso a internet.

Por otro lado, el 74,21\% considera que el punto Vive Digital no siempre satisface sus expectativas en términos de espacio de apoyo para sus labores académicas, a pesar de reconocer en su totalidad (100\%) la inherencia del punto a esta función. Perciben que algunos recursos no funcionan adecuadamente, lo cual se corrobora, por ejemplo, cuando el $69,18 \%$ establece que el software educativo empleado riñe con la actualización de los equipos, debido a la ausencia de licencias renovadas o a retrasos en su adquisición.

En relación con la posibilidad de trabajo colaborativo permitido por el punto, el 75,46\% califica como deficiente esta opción, debido a que los problemas en la conectividad, incluso en los hogares, imposibilitan la promoción de iniciativas concretas de cooperación y hasta la articulación con otras áreas distintas a la Informática. Esto último se evidencia cuando los estudiantes manifiestan que el horario de uso del punto se corresponde con dicho curso.

El siguiente aspecto abordado en la encuesta, a partir del diligenciamiento del cuestionario semiestructurado, referente a la incorporación del punto en las actividades de aula, señala que tan solo el 3,77\% encuentra concreta y efectiva esta sinergia, reconociendo que esta falencia obedece a las limitaciones asociadas a la cobertura y a la ausencia de capacitación en el manejo de TIC, tanto para los docentes como para los estudiantes.

Cuando se consulta a los estudiantes acerca del porcentaje de asociación que perciben entre las labores del punto y sus principales cursos, las respuestas son las siguientes: Informática (96,93\%), Ciencias Sociales (19,02\%), Matemáticas (13,5\%), Ciencias Naturales (11,04\%) e Inglés (4,91\%), lo que confirma lo expresado de la relación punto-Informática y preocupa en términos de Inglés, dado que su influencia en el mundo digital es importante.

Otro aspecto preocupante es que los estudiantes consideran en un 98,11\% que la labor docente no es siempre influyente en su aprendizaje en el punto y que la incorporación tecnológica que éste supone no siempre marca una trascendental diferencia en relación con una clase tradicional (84,28\%), a pesar de las expectativas del 100\% de la población. Aseveraciones en contravía con la misión del Plan Vive Digital.

Adicional a esto, cuando los estudiantes califican de 1 a 5 las funciones del punto, la función de "permitir la participación ciudadana" obtiene el menor puntaje promedio $(2,91)$, situación que también riñe con la labor de inclusión social propuesta por MinTIC a través del punto.

El último aspecto de la encuesta, relacionado con la apropiación social del punto, también pone en evidencia situaciones de déficit complejas. La primera hace alusión a que los estudiantes no encuentran en un 58,49\% asociación entre el Plan Vive Digital y la resolución de los problemas sociales de la región. 
Sumado a esto, el 61,01\% tampoco encuentra relación entre lo que se hace en el punto y la preservación de su identidad rural o campesina, por lo que este espacio es visto más como un escenario de entretenimiento, tecnificación, e incluso a culturación.

En cuanto a las entrevistas realizadas, las afirmaciones contenidas en la Tabla 2 corroboran y complementan lo expuesto en lo observado y en la encuesta:

TABLA 2.

Respuestas más representativas en las entrevistas, por componente

\begin{tabular}{|c|c|}
\hline Componente & Opiniones \\
\hline Brecha digital & $\begin{array}{l}\text {-"Si bien nosotros vivimos en esta región apartada, nuestros gustos tecnológicos son } \\
\text { los mismos de cualquier joven de nuestra generación, de ahí nuestra facilidad de } \\
\text { uso y necesidad de conectividad permanente, a pesar de las limitaciones en TIC y } \\
\text { cobertura". } \\
\text {-"Vemos en las redes sociales una posibilidad de escape de nuestra realidad y de } \\
\text { inserción en el mundo". } \\
\text {-"Se aplaude el esfuerzo del Gobierno con el punto, pero no es suficiente". } \\
\text {-"La cantidad de estudiantes sobrepasa la cantidad de equipos y para aprender } \\
\text { sobre tecnología lo ideal es cacharrear [trabajar] solo”. } \\
\text {-"Es una lástima no poder contar con internet en nuestras casas, pues esto nos } \\
\text { ausenta aún más del desarrollo global”. }\end{array}$ \\
\hline Habitus tecnológico & $\begin{array}{l}\text {-"Sinceramente nosotros usamos más el punto para ocio que para realizar } \\
\text { actividades académicas, pues muchos queremos ser influenciadores”. } \\
\text { “Es que el profesor no entiende que el mundo cambió y que yo aprendo mejor con } \\
\text { juegos que con teoría, y las TIC deben asociarse más a la diversión que a la escuela". } \\
\text {-Me gustan los foros y discusiones virtuales, pero sobre temas de mi interés". } \\
\text {-En realidad, el punto Vive Digital, si bien nos enseña sobre tecnología, está muy } \\
\text { distante de enseñarnos sobre su aplicación en nuestro contexto real”. } \\
\text {-"Yo uso mi tiempo en el punto, cuando es posible y funciona, para construir mi } \\
\text { identidad digital, pero esto como que no interesa mucho a los profesores". }\end{array}$ \\
\hline Alfabetización digital & $\begin{array}{l}\text {-"Sabemos utilizar la red y buscar en ella, pero no sabemos si lo que encontramos es } \\
\text { verdad o mentira, sin embargo, pensamos que todo lo que muestra Google es cierto". } \\
\text {-"Creemos que existe desactualización de los profesores, no tanto en su } \\
\text { conocimiento, sino en el uso de una nueva tecnología y su implementación en la } \\
\text { clase". } \\
\text {-"Es increíble que pocos cursos hayan integrado las TIC al aula". } \\
\text {-"Pienso que se sigue educando igual y se asumen las TIC como una moda a } \\
\text { utilizar". } \\
\text {-"La tecnología es innata para nosotros, así que creo que el punto tiende a } \\
\text { subutilizarse, pues no se profundiza en lo que allí se enseña". }\end{array}$ \\
\hline $\begin{array}{l}\text { Uso, implementación y } \\
\text { apropiación de TIC }\end{array}$ & $\begin{array}{l}\text {-"El punto debería educarnos para transformar nuestro entorno, pero eso no sucede". } \\
\text {-"Asumimos esto como un proceso de cumplimiento gubernamental, desde Bogotá, } \\
\text { más que como un verdadero proceso tendiente a nuestro desarrollo". } \\
\text {-"Si bien el punto nos capacita en lo virtual, nuestros verdaderos problemas siguen } \\
\text { abordándose desde el ejercicio presencial tradicional". } \\
\text {-"No vemos una articulación entre Gobierno, Institución, sociedad y familia, a pesar } \\
\text { que internet y las TIC lo permiten". } \\
\text {-"El Gobierno debería brindar condiciones para educarnos mejor y eso se logra } \\
\text { fortaleciendo el punto. Es que el punto no es malo, simplemente necesita más } \\
\text { atención". } \\
\text {-"Existe en nosotros un problema de identidad, pues no nos sentimos campesinos ni } \\
\text { estamos a la vanguardia de lo digital". }\end{array}$ \\
\hline
\end{tabular}

Fuente: Elaboración propia. 


\section{DiscUSIÓN}

La tercera fase de la investigación no se asume solo como un resultado del ejercicio sino como un plantemiento que amerita su discusión. Por ello, para hablar de una mejora de la relación entre el punto principal del Plan Vive Digital de El Tambo (Cauca) y la comunidad, se propone una estrategia concreta de apropiación social TIC (Proenza y Girard, 2015 y Bacallao, 2018).

Esta estrategia, de índole formativa, permitirá implementar acciones tendentes a la resignificacion de las prácticas digitales (necesidad imperiosa en estos tiempos, según Seale, 2020), en función de fortalecer las costumbres y cultura campesina, con la idea de reconfirmar la identidad de los jóvenes, al intentar relacionar por lo menos un aspecto de su realidad actual con el entorno digital propuesto por el punto, a partir de la generación de contenidos, combinando de esta forma un ecosistema digital (planteado por el Gobierno y la Institución) con un contexto real (vivido por la Institución y sus estudiantes). Al respecto de esta relación holística, Islas y Carranza (2017) hacen un recuento importante de la literatura.

De esta manera, la estrategia propuesta, la cual tiene por nombre "Uso, Incorporación y Apropiación Social de las TIC: El Arte de Cultivar", involucra tres tipos de competencias soportadas en el hacer combinado dentro y fuera del punto (Valencia et al., 2016): 1) Tecnológica: utiliza y promueve el uso de las TIC, a partir de la adquisición de conocimientos y habilidades digitales en el punto Vive Digital; 2) Comunicacional: narra, produce y circula contenidos comunicacionales a través del aprendizaje adquirido en el punto; y 3) Social: pone al servicio del aprendizaje adquirido el saber ancestral (herencia y memoria) asociado con el arte de cultivar, impactando su comunidad y entorno.

Las asignaturas involucradas en el marco del Plan Vive Digital serían: Informática, debido a que es el curso fundamental de apoyo al punto; y Ciencias Sociales, debido a que es el curso que junto con Informática es percibido por los estudiantes como el de mayor relación con el punto y aquel en el que se pueden involucrar la historia, la memoria y la identidad de El Tambo, en términos del fomento del pensamiento crítico desde una mirada socio-humanística.

Esta estrategia social, tecnológica y formativa estaría conformada por cinco fases:

1. Planeación: Se planifica la estructura de la estrategia para su implementación. Se definen las temáticas a abordar (comunes a los dos cursos - se proponen: "costumbres y tradiciones campesinas de El Tambo, Cauca" (componente social) y "afianzamiento del uso de herramientas TIC" (componente informático)), las TIC apropiadas a incorporar (para emplear software como canvas.com, piktochart, etc.), el Capital Humano necesario (tanto de parte de la Institución como de la comunidad) y los canales de publicación y distribución de contenidos (se sugiere la red social Facebook, por ser la más popular).Todo esto confluye en cuatro cursos concretos que transversalizan las temáticas, con foco en el arte de cultivar: 
a. Lo Que Somos: El Arte de Cultivar. Acerca a los jóvenes a las prácticas campesinas, a través del reconocimiento de sus saberes, tradiciones (costumbres, mitos, leyendas e historia popular), herencia cultural, gastronomía, siembra agroecológica y preservación del medio ambiente. Aquí las TIC servirían para explicitar los conocimientos tácitos de la población y construir narrativas digitales (hipertexto) con impacto en la comunidad.

b. Lo Que Oímos: El Arte de Cultivar. Aquí los jóvenes, aprovechando la consola de radio y los micrófonos del punto, adquirirían habilidades y destrezas radiofónicas (tradicionales y digitales), soportadas en lenguajes radiofónicos y producción sonora, las cuales acompañarían a las narrativas construidas. Aquí la tecnología del punto serviría para realizar las grabaciones, editarlas y crear los podcasts, que posteriormente se subirían a la red social.

c. Lo Que Vemos: El Arte de Cultivar. Aquí los estudiantes tendrían la posibilidad de utilizar los elementos audiovisuales del punto, para construir planos, composiciones, imágenes, fotografías, que acompañen lo sonoro y lo narrativo. En este curso las TIC se utilizarían para la elaboración de los guiones y la producción y posproducción audiovisual, mediando para poder compartir los contenidos en la red social.

d. Lo Que Compartimos: El Arte de Cultivar. Este curso serviría para conjuntar lo aprendido en los tres anteriores y dar a conocer dichos aprendizajes en la red social, juntando todos los recursos para crear historias multimediales en un entorno digital accesible por los estudiantes, la Institución, y de manera ideal la comunidad, a través del fomento del uso del punto y sus recursos.

5. Diseño: Los estudiantes, a través de los cursos propuestos, elaboran los prototipos de las piezas comunicativas que se publicarán en la red social. Por ejemplo, para Lo Que Somos: El Arte de Cultivar podrían trabajarse historias o conceptos sobre la identidad rural de los jóvenes y el municipio; para Lo Que Oímos: El Arte de Cultivar se podría generar un guion técnico radial que sirva como sustento para las grabaciones y los podcast; para Lo Que Vemos: El Arte de Cultivar podrían elaborarse conceptos de piezas fotográficas, storytellings o guiones audiovisuales, y para Lo Que Compartimos: El Arte de Cultivar se podría trabajar en la apariencia de la red social o en el diseño de sitios web alternos.

6. Desarrollo: Es la puesta en marcha de la planeación aunada con los componentes del diseño, en función de la elaboración concreta de las piezas comunicativas. Podrían ser: piezas escritas (guiones, noticias, textos, narrativas), piezas radiofónicas (audios, programas cortos), piezas visuales (infografías, posters, retratos), piezas audiovisuales (clips, videos) y piezas multimediales, surgidas de la conjunción de las anteriores.

7. Comunicación: Es la interacción entre los estudiantes, la red social y el público objetivo. Se podría crear una fan page o un foro asociado a Facebook, donde se evidencien y comenten los productos y resultados de la implementación de la estrategia.

8. Socialización: Es la presentación de esta propuesta y sus componentes al personal del Plan Vive Digital, a la Institución Educativa y a la comunidad de El Tambo (Cauca). 


\section{CONCLUSIONES}

Se establece que existen notables diferencias entre lo que se espera de la incorporación, uso y apropiación social de las Tecnologías de la Información y la Comunicación [TIC], en el marco del Plan Vive Digital del Ministerio de las Tecnologías de la Información y las Comunicaciones (MinTIC), en el municipio de El Tambo (Cauca, Colombia), y lo que realmente se logra desarrollar en la institución objeto de estudio por ser punto principal de acción del Plan.

En términos del objetivo propuesto, se identifica el alcance a través de los siguientes hechos concretos: el Gobierno colombiano ha llevado a cabo una apuesta importante desde la ciencia y la tecnología por efectivizar el papel de las TIC en perspectiva de desarrollo, siendo el Plan Vive Digital un ejemplo de ello. Y en este marco, se pueden encontrar una serie de acciones, desde los niveles estratégicos hasta los operativos, en los que el Estado desarrolla despliegues que propenden por la disminución de las brechas digitales en torno a programas de alfabetización.

Sin embargo, en el contexto de las regiones colombianas, y específicamente en el objeto de estudio, se evidencia una latente inequidad y desarticulación con la propuesta emanada desde la intención estatal, pues se sustenta esta aifrmación en el escaso conocimiento que tienen las poblaciones rurales sobre las formas de incorporar la tecnología en los procesos de enseñanza-aprendizaje; así como en las deficientes infraestructuras locativas y tecnológicas, que hacen mucho más pronunciado el problema aquí evidente.

Esto limita la posibilidad de incorporación en el aula de este anhelado entorno digital y, por ende, las tecnologías para el aprendizaje pasan por la reflexión sobre la equidad, en tanto no tienen las mismas implicaciones los entornos virtuales de aprendizaje desarrollados en contextos socioeconómicos favorables o no.

Todo lo anterior riñe con el interés del joven estudiante por sentirse partícipe del mundo tecnológico, pues éste se ve seducido por pertenecer a un espacio de interacción digital global, en el cual sus necesidades de consumo mediático (y no solamente las redes sociales) le llevan a pretender una inserción inmediata y protagónica en este nuevo marco de acción, siendo, desde su imaginario, el Plan Vive Digital el escenario propicio para lograrlo. Sin embargo, esto no ocurre de esta manera.

Es por esta razón, que la estrategia formativa propuesta intenta articular el interés y motivación del estudiante por lo digital, con la misión del plan, con la labor de la institución formadora como punto Vive Digital y con las necesidades, intereses y posibilidades reales de apropiación de la comunidad, pues solo de esta manera se logrará un acercamiento efectivo a la sociedad de la información y el conocimiento.

\section{REFERENCIAS}

Andrade, L., Carbonell, X. y López, V. M. (2018). Variables sociodemográficas y uso problemático en adolescentes ecuatorianos. Health and Addictions, 19(1), 1-10. http:// dx.doi.org/10.21134/haaj.v19i1.391 
Bacallao-Pino, L. M. (2018). What power? Social representations of ICTs' appropriation for community empowerment in Latin American social movements. Semiotica, 2018(223), 177-197. https://doi.org/10.1515/sem-2017-0013

Barraquero, A. (2009). Latinoamérica en el paradigma participativo de la comunicación para el cambio. Málaga: Universidad de Málaga.

Barraquero, A. (2006). Comunicación y cambio social en España. Balance, escenarios y perspectivas. Málaga: Universidad de Málaga.

Barraquero, A. y Ángel, A. (2014). La producción académica sobre comunicación, desarrollo y cambio social. Signo y Pensamiento, 34(67), 30-57. https://doi.org/10.11144/ Javeriana.syp34-67.pacd

Barón, L. y Gómez, R. (2014). Más que teclas y pantallas: Acceso público a TIC en Colombia. Santiago de Cali: Universidad Icesi. Disponible en http://eduteka.icesi.edu.co/ articulos/investigacion-teclas-pantallas

Bawden, D. (2002). Revisión de los conceptos de alfabetización informacional y alfabetización digital. Anales de Documentación, (5), 361-408. Disponible en https://revistas.um.es/analesdoc/article/view/2261

Cabrera, D. (2006). Lo tecnológico y lo imaginario. Madrid: Biblos. Recuperado de https:// imaginariosyrepresentaciones.files.wordpress.com/2016/04/lo_tecnologico_y_lo_imaginario-_las_nuev1.pdf

Calderón, M. J. y Acuña, M. J. (2017). Conectividad rural y cambio social: los infocentros comunitarios de Ecuador. Revista Publicando, 4(11), 190-207. Recuperado de https:// revistapublicando.org/revista/index.php/crv/article/view/520/pdf_347

Casillas, M. A. y Ramírez, A. (2018). El habitus digital: una propuesta para su observación. En: R. Castro y H. J. Suárez (Ed.), Pierre Bourdieu en la sociología latinoamericana. El uso de campo y habitus en la investigación (pp. 317-341). Cuernavaca: Universidad Nacional Autónoma de México-UNAM.

Castells, M. (2009). La apropiación de las tecnologías. Cultura juvenil en la era digital. Telos. Cuadernos de Comunicación e Innovación, (81), 111-113. Disponible en https:// telos.fundaciontelefonica.com/archivo/numero081/la-apropiacion-de-las-tecnologiasla-cultura-juvenil-en-la-era-digital/

Chaparro, F. (2003). Apropiación social del conocimiento, aprendizaje y capital social. Simposio Internacional sobre Ciencia y Sociedad, Universidad de Antioquia, Medellín, Colombia.

Cobo, J. C. (2009) El concepto de tecnologías de la información. Benchmarking sobre las definiciones de las TIC en la sociedad del conocimiento. RZER-Revista de Estudios de Comunicación, 14(27), 295-318. Disponible en https://www.ehu.eus/ojs/index.php/ Zer/article/view/2636/2184

Chong, A. (2011). Conexiones de desarrollo: el impacto de las TIC. Washington, D.C.: BID-Banco Interamericano de Desarrollo.

Chong, B., Aguilar, G., Ornelas, J., Morales, J. y Torre, S. (2011). Enrédate. Tecnologías comunitarias. Réplica de un proyecto sobre apropiación de las TIC en sectores marginados. En: C. M. Krohling, T., Tufte y M. J., Vega (Ed.), Trazos de otra comunicación en América Latina: prácticas comunitarias, teorías y demandas sociales (pp. 82-93). Barranquilla: Universidad del Norte. Disponible en http://hdl.handle.net/10584/1192 
Echeverría, J. (2008). Apropiación social de las Tecnologías de la Información y la Comunicación. Revista Iberoamericana de Ciencia, Tecnología y Sociedad-CTS, 4(10), 171-182. Disponible en http://www.revistacts.net/files/Volumen\%204\%20-\%20 N\%FAmero\%2010/doss07.pdf

Escobar, A. (2003). Globalización, desarrollo y modernidad. Medellín: Organización de Estados Iberoamericanos-OEI.

Escobar, J. M. (2014). ¿Sociedad de la Información? Miradas sobre la apropiación social de las tecnologías. Santiago de Cali: Programa Editorial Universidad Autónoma de Occidente.

Escosteguy, A. C., Bianchini, A. e Ribas, J. V. (2018). A noção de espaço na apropriação de tecnologias de comunicação no rura contemporáneo. Revista FAMECOS-Midia, Cultura e Tecnologia, 25(2), 1-19. http://dx.doi.org/10.15448/1980-3729.2018.2.28325

Espinoza, N. (2010). La brecha digital. Avances para su superación en Venezuela. Revista Iberoamericana de Ciencia, Tecnología y Sociedad-CTS, 1, 1-16. Recuperado de http://www.revistacts.net/wp-content/uploads/2010/08/espinoza_edit.pdf

Fernández, J. (2012). La tercera y definitiva brecha digital. Telos: Cuadernos de Comunicación e Innovación, (91), 6-8. Disponible en https://telos.fundaciontelefonica.com/ archivo/numero091/la-tercera-y-definitiva-brecha-digital/

Gumucio, A. (2011). Comunicación para el cambio social: clave del desarrollo. Signo y Pensamiento, 30(58), 26-39. Recuperado de https://revistas.javeriana.edu.co/index. php/signoypensamiento/article/view/2454/1728

Hernández, R., Fernández, C. y Baptista, M. P. (2015). Metodología de la investigación (6 ed.). México, D.F.: McGraw-Hill. Recuperado de https://www.uca.ac.cr/wp-content/ uploads/2017/10/Investigacion.pdf

Islas, C. y Carranza, M. R. (2017). Ecosistemas digitales y su manifestación en el aprendizaje: análisis de la literatura. RED. Revista de Educación a Distancia, (55), 1-13. http://dx.doi.org/10.6018/red/55/9

Lemus, M. (2017). De accesos e igualaciones: apropiación de TIC por jóvenes en el marco del Programa Conectar Igualdad. Ciencia, Docencia y Tecnología, 28(54), 150-187. Disponible en http://www.pcient.uner.edu.ar/cdyt/article/view/259/285

Martínez, L. A. (2007). La observación y el diario de campo en la definición de un tema de investigación. Perfiles Libertadores, 1(1), 73-80. Recuperado de https://www. ugel01.gob.pe/wp-content/uploads/2019/01/1-La-Observaci\%C3\%B3n-y-el-Diario-decampo-07-01-19.pdf

Méndez, C. E. (2015). Metodología: diseño y desarrollo del proceso de investigación (4 ed.). México, D.F.: Grupo Noriega Editores.

Morduchowicz, R. (2013). Los adolescentes y las redes sociales. Buenos Aires: Fondo de Cultura Económica.

ONU. (junio 19, 2019). ¿Qué puede hacer la tecnología en beneficio del desarrollo? Noticias ONU. [Boletín]. Recuperado de https://news.un.org/es/story/2019/06/1457461

ONU. CEPAL. (2018). Segundo informe anual sobre el progreso y los desafios regionales de la Agenda 2030 para el Desarrollo Sostenible en América Latina y el Caribe. Santiago de Chile: CEPAL. Disponible en https://repositorio.cepal.org/bitstream/handle/11362/43415/5/S1800380_es.pdf 
ONU. PNUD. (2020). ¿Qué son los objetivos de desarrollo sostenible? [Online]. Recuperado de https://www.undp.org/content/undp/es/home/sustainable-development-goals. html

ONU. PNUD Colombia. (septiembre 28, 2015). ¿Cómo le fue a Colombia con los objetivos de desarrollo del milenio? [Online]. Recuperado de http://www.co.undp.org/content/colombia/es/home/presscenter/articles/2015/09/28/-c-mo-le-fue-a-colombia-con-los-odm-. html

Pathak-Shelat, M. y DeShano, C. (2014). Digital youth cultures in small town and rural Gujarat, India. New Media \& Society, 16(6), 983-1001. https://doi. org/10.1177/1461444813496611

Patton, M. Q. (2014). Qualitative research \& evaluation methods: Integrating theory and practice (4 ed.). Thousand Oaks: Sage Publications.

Paulhiac, J. C. y Ortega, A. J. (2019). Uso y apropiación de las TIC: una exploración del acceso a los cibercafés y Kioskos Vive Digital en comunidades rurales. Análisis, 51(95), 289-318. https://doi.org/10.15332/21459169.4456

Paz, P. C. (2015). Diagnóstico de la página Web de la Asociación de Cabildos Indígenas del Norte del Cauca-Colombia. Razón y Palabra, 20(1-92), 1701-1748. Disponible en https://www.revistarazonypalabra.org/index.php/ryp/article/view/886

Pérez, A. (2015). Alfabetización digital y competencias digitales en el marco de la evaluación educativa: estudio en docentes y alumnos de educación primaria en Castilla y León. Salamanca: Universidad de Salamanca.

Prensky, M. (2001). Nativos digitales / emigrantes digitales de “On the Horizon”. Bingley: MCB University Press.

Proenza, F. J. \& Girard, B. (2015). The impact of public access to telecenters: Social appropriation of ICT by chilean women. Cambridge: MIT Press.

Quinchoa, W. J. (2011). Apropiación y resistencia social de las TIC en el resguardo indígena de Puracé, Cauca, Colombia. Revista Iberoamericana de Ciencia y Tecnología, 6(18), 241-258. Disponible en http://www.revistacts.net/contenido/numero-18/walterjulian-quinchoa-cajas/

República de Colombia. DANE. (2019). Resultados Censo Nacional de Población y Vivienda 2018. Popayán, Cauca. [Online]. Recuperado de https://www.dane.gov.co/files/censo2018/informacion-tecnica/presentaciones-territorio/190814-CNPV-presentacionResultados-generales-Cauca.pdf

República de Colombia. DANE. (2018). Estadísticas por tema. Demografía y población. [Online]. Recuperado de https://www.dane.gov.co/index.php/estadisticas-por-tema/ demografia-y-poblacion

República de Colombia. MinTIC. (agosto 3, 2020). Acerca del MinTIC. [Online]. Recuperado de https://www.mintic.gov.co/portal/inicio/Ministerio/Acerca-del-MinTIC/

República de Colombia. MinTIC. (2018). Vive Digital. [Online]: Recuperado de https:// mintic.gov.co/portal/vivedigital/612/w3-channel.html

República de Colombia. MinTIC. (2017). Kioscos Vive Digital del Departamento del Cauca. [Online]. Recuperado de https://www.datos.gov.co/Educaci-n/Kioscos-Vive-digitaldel-Departamento-del-Cauca/4sk6-8wqf 
República de Colombia. MinTIC. (30 de junio de 2009). Por la cual se definen Principios y conceptos sobre la sociedad de la información y la organización de las Tecnologías de la Información y las Comunicaciones -TIC-, se crea la Agencia Nacional del Espectro y se dictan otras disposiciones. [Ley 1341]. DO: 47.426. Recuperado de https://mintic. gov.co/portal/604/articles-8580_PDF_Ley_1341.pdf

Rueda, R. (2005). Apropiación social de las tecnologías de la información: ciberciudadanías emergentes. Tecnología y Comunicación Educativas, 20(41), 19-32. Disponible en http://investigacion.ilce.edu.mx/tyce/41/art2.pdf

Sachs, W. (1996). Diccionario del desarrollo. Una guía del conocimiento como poder. Lima: Proyecto Andino de Tecnologías Campesinas-PRATEC. Disponible en https:// www.uv.mx/mie/files/2012/10/SESION-6-Sachs-Diccionario-Del-Desarrollo.pdf

Seale, J. (2020). Improving accessible digital practices in higher education: Challenges and new practices for inclusion. Cham: Palgrave Pivot.

Valencia, T., Serna, A., Ochoa, S., Caicedo, A. M., Montes, J. A. y Chávez, J. D. (2016). Competencias y estándares TIC desde la dimensión pedagógica: una perspectiva desde los niveles de apropiación de las TIC en la práctica educativa docente. Cali: Pontificia Universidad Javeriana-PUJ y Organización de las Naciones Unidas para la Educación, la Ciencia y la Cultura-UNESCO. Disponible en http://www.unesco.org/ new/fileadmin/MULTIMEDIA/FIELD/Santiago/pdf/Competencias-estandares-TIC. pdf

Vásquez, F. E. (2012). Educación y tecnología: relación vital en la sociedad del conocimiento. En: Corporación Colombia Digital (Ed.). Aprender y educar con las tecnologías del Siglo XXI (pp. 71-82). Bogotá, D.C.: Corporación Colombia Digital. Disponible en https://libros.metabiblioteca.org/bitstream/001/502/1/Libro-Aprender-y-Educar.pdf

Vásquez-Rizo, F. E. y Gabalán-Coello, J. (2011). Implementación de un modelo de administración de capital humano en un grupo de investigación. Profesional de la Información, 20(5), 516-526. http://dx.doi.org/10.3145/epi.2011.sep.05

Vásquez, F. E., Rodríguez, J. V. y Gómez, J. A. (2019). La gestión de información para medir la capacidad investigadora en una institución de educación superior. Revista Espacios, 40(8), 18-30. Disponible en https://revistaespacios.com/a19v40n08/19400818. html

Villacrés, J. R. (2016). Incidencia del uso de las Tecnologías de Información y Comunicación (TIC) en los hábitos y costumbres de jóvenes de cultura Kichwa en Ecuador. Asian Journal of Latin American Studies, 29(1), 109-131. Recuperado de http://www. ajlas.org/v2006/paper/2016vol29no105.pdf

Winocur, R. (2013). Una revisión crítica de la apropiación en la evaluación de los programas de inclusión digital. En, S. Morales y M. Loyola, (coord.), Nuevas perspectivas en los estudios de comunicación: la apropiación tecno-mediática (pp. 53-64). Buenos Aires: Imago Mundi.

María Estefanía Orozco-García es docente Tiempo Completo de la Fundación Universitaria de Popayán (Colombia). Magíster en Comunicación y Especialista en Comunicación Organizacional de la Universidad Autónoma de Occidente (Colombia). https://orcid. org/0000-0002-0856-8472 
Fredy Eduardo Vásquez-Rizo es docente Tiempo Completo de la Universidad Autónoma de Occidente (Colombia). PhD en Gestión de la Información y de la Comunicación en las Organizaciones de la Universidad de Murcia (España). Magíster en Ciencias de la Información y Administración del Conocimiento del Instituto Tecnológico de Estudios Superiores de Monterrey-ITESM (México). http://orcid.org/0000-0003-1398-6174

Jesús Gabalán-Coello es asesor de Rectoría de la Universidad Católica de Pereira (Colombia). PhD en Medición y Evaluación en Educación de la Universidad de Montreal (Canadá). Magíster en Ingeniería con énfasis en Ingeniería Industrial de la Universidad del Valle (Colombia). 